\title{
Reconfigurable intelligent surfaces assisted wireless communication networks: ergodic capacity and symbol error rate
}

\author{
Dinh-Thuan Do, Chi-Bao Le \\ Faculty of Electronics Technology, Industrial University of Ho Chi Minh City, Ho Chi Minh City, Vietnam
}

\section{Article Info}

\section{Article history:}

Received Apr 19, 2021

Revised Nov 15, 2021

Accepted Nov 25, 2021

\section{Keywords:}

Ergodic capacity

Reconfigurable intelligent surfaces

Symbol error rate

\begin{abstract}
By enabling reconfigurable intelligent surfaces (RIS), we can deploy intelligent reflecting signals from the base station to destinations. Different from traditional relaying system, RIS relies on programmable metasurfaces and mirrors to improve system performance of destinations. We derive the formulas of main system performance metrics such as ergodic capacity and symbol error rate (SER). Based on types of modulation, we need to demonstrate other parameters which make influence to system performance. We show analytically that the number of reflecting elements along with the transmit power at the source can improve system performance. Moreover, we check the exactness of derived expressions by matching Monte-Carlo with analytical simulations. Finally, we find the best performance can be achieved at specific parameters and results are verified by explicit simulations.
\end{abstract}

This is an open access article under the CC BY-SA license.

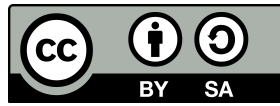

\section{Corresponding Author:}

Dinh-Thuan Do

Faculty of Electronics Technology, Industrial University of Ho Chi Minh City

12 Nguyen Van Bao Street, Go Vap District, Ho Chi Minh City 700000, Vietnam

Email: dodinhthuan@iuh.edu.vn

\section{INTRODUCTION}

To implement next-generation wireless communications, one can deploy reconfigurable intelligent surfaces (RISs) to enable current systems with solid requirements such as low cost, high energy-efficiency and higher bandwidth efficiency. RISs exhibit their appealing ability by adjusting the propagation of the electromagnetic waves [1]-[3]. By integrating of passive and reflecting units, the RIS-aided systems can adjust phases and amplitudes independently for the incident signals. Further, RIS provides a massive connections and exploits a full-duplex scheme to reflect signals to destinations. As main advances, the RIS shows benefits when we compare it with the contemporary relaying systems. First, to avoid power-hungry radio frequency processing, the RIS is deployed as a passive device and thus less energy is acquired to conduct the reflection. Second, to introduce low-cost deployment, the RIS can be easily deployed on various environmental objects, for example building facades, street signs and advertisement boards [4]. Furthermore, in perceptive of information transfer, the reflection pattern is implemented at the RIS to improve system performance [5]-[7].

Specifically, Rehman et al. [8] studied expressions of the outage probability and average sum-rate by assuming that the RIS-aided system is optimized when the system can achieve the highest instantaneous end-to-end signal-to-noise ratio (SNR). RIS thus is deployed to improve the current systems in terms of interference cancellation, secure transmission, wireless coverage, throughput enhancement, wireless information and power transfer. Importantly, Pan et al. [9] proposed the system to allow the angle of reflection of each RIS 
element can be adjusted to enhance the coverage performance significantly. While the amplify-and-forward (AF) shows high complexity in signal processing, and works in full-duplex with degraded performance due to self-interference, RIS only reflects the received signals passively which is prominent compared to the conventional relaying systems. As a result, RIS-aided systems enhance the system energy efficiency (EE) with high spectral efficiency (SE) without additional transmission power consumption. By combining both nonorthogonal multiple access (NOMA) [10]-[14] and RIS, the NOMA-RIS is proposed to improve the system performance in specific system metrics.

Research explored RIS system by combining the phase shifts at the RIS and the joint optimization of the beamformer at the base station, then system performance can be optimized [15], [16]. In [17]-[21], various system models are presented to demonstrate benefits of RIS. For example, in [17], Jiang and Shi considered the assistance of a multi-element RIS to boost the performance of over-the-air computation. Research presented secure transmission in the presence of eavesdroppers [18]-[20]. They considered the system that multi-antenna base station servers multiple single-antenna legitimate users with the assistance of a multi-element RIS. Yan et al. [21] proposed that a multi-element RIS is required to assist the primary communication between a multi-antenna base station and a single-antenna user. Motivated by recent studies [18]-[21], this article aims to consider two main system metrics, i.e. ergodic capacity and symbol error rate for point-to-point RIS-aided system.

The main notations of this paper is shown as follows: $\mathbb{E}[\bullet]$ denotes expectation operation; $f_{X}(\bullet)$ and $F_{X}(\bullet)$ denote the probability density function (PDF) and the cumulative distribution function (CDF) of a random variable $X ; G_{p, q}^{m, n}(\bullet \bullet \bullet)$ denotes the Meijer-G function of a single variable; $\Gamma(\bullet)$ is the Gamma function; $\gamma(\bullet, \bullet)$ is the lower incomplete Gamma function; $\mathcal{Q}(\bullet)$ is the Gaussian error function.

\section{SYSTEM MODEL}

We consider the downlink from the base station (BS) which is required to serve a destination (D) with the help of RIS, shown in Figure 1. In particular, the point-to-point RIS-assisted wireless system in this scenario is studied with single-antenna design for BS and D nodes, while $K$ metasurfaces is required at RIS. We represent the baseband equivalent fading channels between the BS and the $k$ th metasurface of the RIS, $\bar{h}_{k}$. In the second hop, the channel between the $k$ th metasurface and node $\mathrm{D}$ is denoted as $\bar{g}_{k}$. We assume characteristic of channels such as independent, identical and slowing varying.

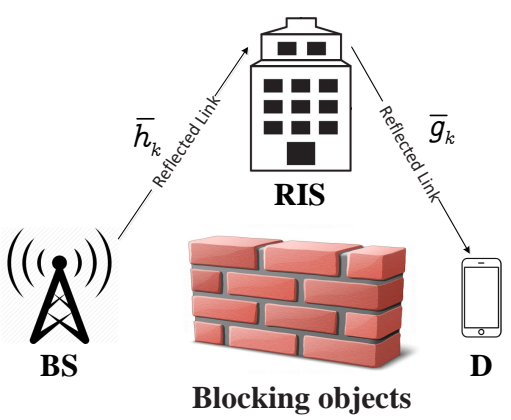

Figure 1. The point-to-point RIS-assisted system

We denote $P_{S}$ ass the normalized transmission powers at the BS. The received signal at user D is given by:

$$
\bar{y}=\sqrt{P_{S}} \sum_{k=1}^{K} \bar{h}_{k} \bar{g}_{k} v_{k} \bar{x}+\omega
$$

where the metasurface has $v_{k}=\left|v_{k}\right| \exp \left(j \bar{\theta}_{k}\right), \bar{\theta}_{k}$ stands for the phase shift related to $k$ th reflective units in the RIS and $\omega$ represents the additive white Gaussian noise (AWGN) and such a noise is considered as a zero-mean complex Gaussian (ZMCG) process with variance equal $N_{0}$. In this case, unit power is assumed for signal $x$, i.e, $\mathbb{E}\left\{|x|^{2}\right\}=1$. 
In our study, we assume $\left|v_{k}\right|=1$ which is in line with real deployment [22]. It is assumed that the RIS has perfect knowledge of the phase of $\bar{h}_{k}, \bar{\theta}_{\bar{h}_{k}}$ and the one $\bar{g}_{k}, \bar{\theta}_{\bar{g}_{k}}$, and selects the optimal phase shifting, i.e.

$$
\bar{\theta}_{k}=-\left(\bar{\theta}_{\bar{h}_{k}}+\bar{\theta}_{\bar{g}_{k}}\right) \text {. }
$$

To easier manipulations, we denote $\mathcal{A}=\sum_{k=1}^{K}\left|\bar{h}_{k}\right|\left|\bar{g}_{k}\right|$ as baseband equivalent channel coefficient. Then, we can compute the received signal as (3).

$$
\bar{y}=\mathcal{A} x+\omega .
$$

To further achieve other system metrics, we need to obtain the instantaneous the signal-to-noise-ratio (SNR) as (4).

$$
\bar{\gamma}=\frac{|\mathcal{A}|^{2} P_{S}}{N_{0}}
$$

We can rewrite (4) as:

$$
\bar{\gamma}=|\mathcal{A}|^{2} \rho_{S},
$$

in which $\rho_{S}=P_{S} / N_{0}$ represents for the BS in term of signal-to-noise radio (SNR). It is better examine important system performance at destination and the other system performance metrics can be determined by exploiting such SNR. We expect that high SNR leads to better system performance.

\section{ANALYSIS OF ERGODIC CAPACITY}

Since ergodic capacity plays an important role to evaluate system performance, we derive a closedform expression for the ergodic capacity (EC) as (6).

$$
\begin{aligned}
\bar{C} & =\mathbb{E}[\log (1+\bar{\gamma})] \\
& =\int_{0}^{\infty} \ln (1+x) f_{\bar{\gamma}}(x) d x .
\end{aligned}
$$

In this step, $\ln (1+x)$ can be formulated with the help of [23, Eq. (8.4.6.5)]:

$$
\ln (1+x)=G_{2,2}^{1,2}\left(x \mid \begin{array}{l}
1,1 \\
1,0
\end{array}\right),
$$

where $G_{p, q}^{m, n}(\bullet \mid \bullet)$ denotes the Meijer-G function of a single variable. So, we can express the PDF and CDF of $\bar{\gamma}$ define in (6) as [24, Eq. (24)], [24, Eq. (25)]:

$$
f_{\bar{\gamma}}(x)=\frac{x^{(a-1) / 2}}{2 b^{a+1} \Gamma(a+1) \rho_{S}^{(a+1) / 2}} \exp \left(-\frac{1}{b} \sqrt{\frac{x}{\rho_{S}}}\right),
$$

and

$$
F_{\bar{\gamma}}(x)=\frac{1}{\Gamma(a+1)} \gamma\left(a+1, \frac{1}{b} \sqrt{\frac{x}{\rho_{S}}}\right)
$$

where $a=\frac{K \pi^{2}}{\left(16-\pi^{2}\right)}-1$ and $b=\frac{8}{\pi}-\frac{\pi}{2}, \Gamma(\bullet)$ is the Gamma function and $\gamma(\bullet, \bullet)$ is the lower incomplete Gamma function.

With the aid of [25, Eq. (8.350.1)], 9) can be claimed by (10).

$$
F_{\bar{\gamma}}(x)=\frac{1}{\Gamma(a+1)} \sum_{l=0}^{\infty} \frac{(-1)^{l} x^{(a+l+1) / 2}}{l !(a+l+1) \rho_{S}^{(a+l+1) / 2} b^{a+l+1}} .
$$

Then, substituting (8) and (7) into (6), the ergodic capacity can be expressed as (11). 


$$
\begin{aligned}
\bar{C} & =\int_{0}^{\infty} G_{2,2}^{1,2}\left(x \mid \begin{array}{c}
1,1 \\
1,0
\end{array}\right) f_{\bar{\gamma}}(x) d x \\
& =\frac{1}{2 b^{a+1} \Gamma(a+1) \rho_{S}^{(a+1) / 2}} \int_{0}^{\infty} x^{(a-1) / 2} G_{2,2}^{1,2}\left(x \mid \begin{array}{c}
1,1 \\
1,0
\end{array}\right) \exp \left(-\frac{1}{b} \sqrt{\frac{x}{\rho_{S}}}\right) d x
\end{aligned}
$$

Let $t=\sqrt{x} \rightarrow t^{2}=x \rightarrow 2 t d t=d x$ and by applying the [26, Eq. (3.3.12)], the closed-form solution of $\bar{C}$ for the ergodic capacity can be determined as (12).

$$
\begin{aligned}
& \bar{C}=\frac{1}{b^{a+1} \Gamma(a+1) \rho_{S}^{(a+1) / 2}} \int_{0}^{\infty} t^{(a+1)-1} \exp \left(-\frac{t}{b \sqrt{\rho_{S}}}\right) G_{2,2}^{1,2}\left(t^{\frac{2}{1}} \mid \begin{array}{l}
1,1 \\
1,0
\end{array}\right) d t \\
& =\frac{2^{a}}{\sqrt{\pi} \Gamma(a+1)} G_{4,2}^{1,4}\left(4 b^{2} \rho_{S} \mid \begin{array}{c}
-a / 2,(1-a) / 2,1,1 \\
1,0
\end{array}\right) \text {. }
\end{aligned}
$$

\section{SYMBOL ERROR RATE}

Let denote $\alpha$ and $\beta$ as constants. In particular, the modulation types depend on values of $\alpha$ and $\beta$. We treat the binary phase-shift keying (BPSK) modulation corresponding to $\alpha=1, \beta=2$. If values are $\alpha=2$, $\beta=1$, they represent for quadrature phase shift keying (QPSK) and 4-quadrature amplitude modulation (4$\mathrm{QAM})$ in [27], $\mathcal{Q}(\bullet)$ is the Gaussian error function. For the RIS-aided point-to-point system, the Symbol Error Rate (SER) need be computed as [27]:

$$
\begin{aligned}
\mathcal{S} & =\alpha E\{\mathcal{Q}(\sqrt{\beta \bar{\gamma}})\} \\
& =\frac{a}{2 \pi} \int_{0}^{\infty} F_{\bar{\gamma}}\left(\frac{x^{2}}{b}\right) e^{-\frac{x^{2}}{2}} d x \\
& \stackrel{\triangleq}{\stackrel{\triangleq}{=} x^{2} / b} \frac{\alpha \sqrt{\beta}}{2 \sqrt{2 \pi}} \int_{0}^{\infty} \frac{e^{-\frac{\beta}{2} x}}{\sqrt{x}} F_{\bar{\gamma}}(t) d t .
\end{aligned}
$$

substituting (10) into (9), the SER of RIS-aided system can be expressed as (14).

$$
\begin{aligned}
\mathcal{S} & =\frac{\alpha \sqrt{\beta}}{2 \sqrt{2 \pi}} \int_{0}^{\infty} \frac{e^{-\frac{\beta}{2} x}}{\sqrt{x}} F_{\bar{\gamma}}(t) d t \\
& =\sum_{l=0}^{\infty} \frac{\alpha \sqrt{\beta}(-1)^{l}}{l ! 2 \sqrt{2 \pi} \Gamma(a+1)(a+l+1) \rho_{S}^{(a+l+1) / 2} b^{a+l+1}} \int_{0}^{\infty} e^{-\frac{\beta}{2} x} x^{(a+l) / 2} d t .
\end{aligned}
$$

We then use the result from [25, Eq. (3.351.3)], the closed-form expression can be obtained to indicate the SER performance. In particular, the expression of $\mathcal{S}$ can be achieved as (15).

$$
\mathcal{S}=\sum_{l=0}^{\infty} \frac{\alpha(-1)^{l} 2^{(a+l) / 2} \beta^{(-a-l) / 2} \Gamma((a+l+1) / 2)}{l ! \sqrt{2 \pi} \Gamma(a+1)(a+l+1) \rho_{S}^{(a+l+1) / 2} b^{a+l+1}} .
$$

Remark: As our observation, (15) depends on values of both $\alpha$ and $\beta$. Therefore, by adjusting the modulation type, we can obtain different performance. We expect to compare the SER performance by comparing two types, i.e. BPSK and QPSK. Further, the SNR at the BS plays key role to indicate improvement of SER since (15) also contains $\rho_{s}$. 


\section{NUMERICAL RESULTS}

This section is conduced to verify expressions obtained in the previous sections. Monte Carlo simulations are conducted to examine exactness of mathematical expressions of performance analysis. We focus on the RIS-assisted wireless system by examining these metrics such as ergodic capacity and SER. Monte-Carlo results are performed by run of $10^{7}$ independent channel realizations.

Figure 2 shows the ergodic capacity performance when we change the number of metasurfaces $K$. As our observation, $K=100$ shows the corresponding ergodic capacity as the highest case among five cases. It can be seen that the analytical results are matched well with Monte Carlo simulations in the whole range of SNR. We also observe that the ergodic capacity increases by increasing SNR at the BS $\rho_{s}$. This is because the end-to-end SNR depends on SNR at the BS, then the corresponding ergodic capacity can be improved at high SNR $\rho_{s}$ region. The performance gaps among five cases are the same in whole range of $\rho_{s}$.

In Figure 3, the ergodic capacity can be enhanced at higher number of metasurfaces $K$ of the RIS. It can be seen clearly the ergodic capacity only increase very fast when $K$ changes from 0 to 400 . After this point, the ergodic capacity just increase slightly. The ergodic capacity performance of RIS-assisted system for the destination is compared with set of SNR at the BS, i.e. $\rho_{s}=20,30,40,50$. We observe that with the increase of $\rho_{s}$ and $K$, the ergodic capacity performance of the considered system is improved significantly at low region of SNR. Therefore, the design of many metasurfaces $K$ is unnecessary.

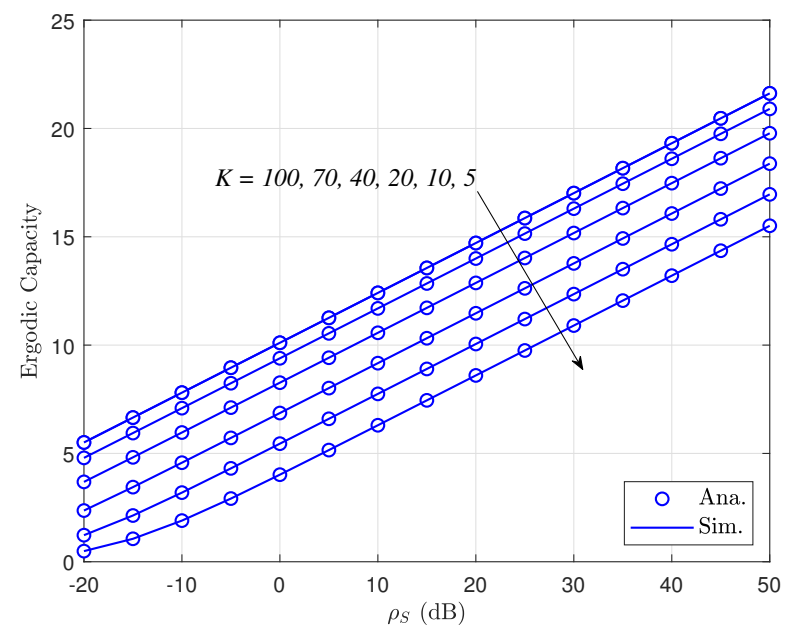

Figure 2. Increasing SNR to look at curves of ergodic capacity

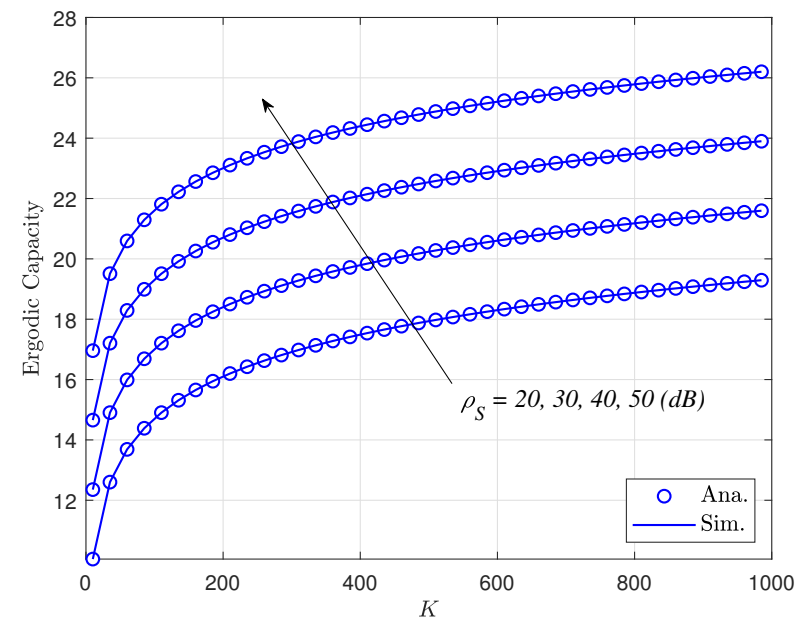

Figure 3. The number of meta-surface makes influence to ergodic capacity 
Figure 4 demonstrates SER performance for the case of BPSK modulation when we vary the SNR at the BS $\rho_{s}$ from $-40 \mathrm{~dB}$ to $10 \mathrm{~dB}$. It can be seen clearly that SER can be improved significantly at the range of $\rho_{s}$ from $-40 \mathrm{~dB}$ to $-10 \mathrm{~dB}$. The main reason is that the (15) depends on $\rho_{s}$. Moreover, the best SER performance can be reported as $K=20$. We can conclude that by designing more metasurfaces $K$ at the RIS, we can achieve good performance in term of SER. Similarly, Figure 5 shows similar performance for the case of QPSK modulation.

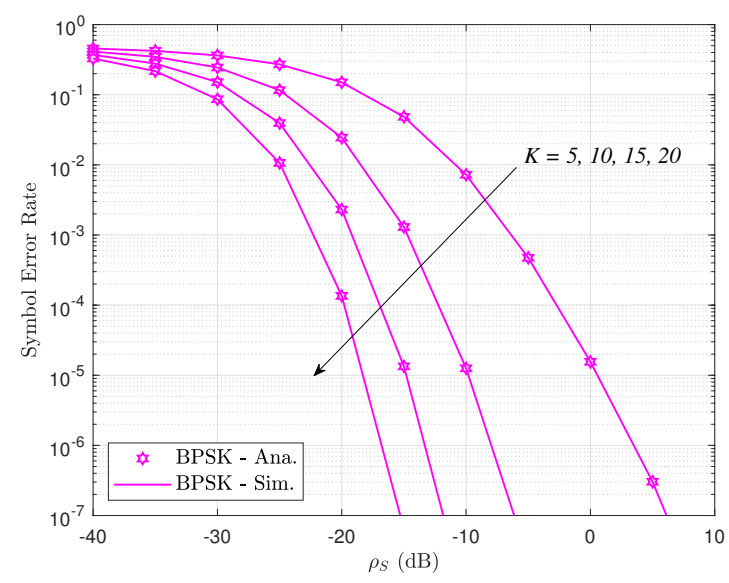

Figure 4. SER versus the SNR at the BS using BPSK

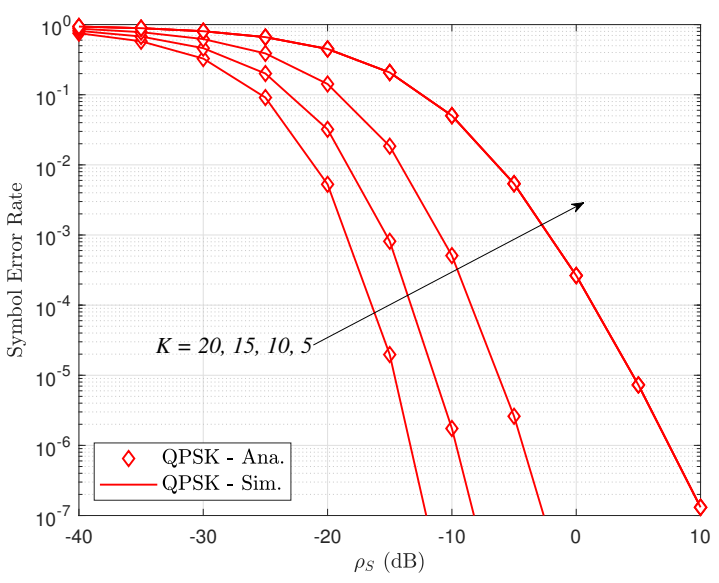

Figure 5. SER versus the SNR at the BS using QPSK

\section{CONCLUSION}

We considered the wireless system by enabling RIS at the downlink. We examine the system performance at two main system metrics, i.e. ergodic capacity and SER. We derived closed-form expressions of ergodic capacity and SER. Based on these derivations, we find that reflecting coefficient $K$ of the RIS that maximizes the the system performance at reasonable value of transmit SNR at the base station. Simulations showed that the main parameters such as transmit SNR at the base station is found as main controlling parameters compared with the number of metasurfaces. The numerical results indicate that the ergodic capacity still has limitations although we increase the number of metasurfaces at RIS.

\section{REFERENCES}

[1] M. Di. Renzo et al., "Smart radio environments empowered by reconfigurable AI meta-surfaces: An idea whose time has come," EURASIP Journal on Wireless Communications and Networking, vol. 2019, no. 1, pp. 1-20, May 2019, doi: 10.1186/s13638-0191438-9.

[2] C. Liaskos, S. Nie, A. Tsioliaridou, A. Pitsillides, S. Ioannidis, and I. Akyildiz, "A new wireless communication paradigm through software controlled metasurfaces," in IEEE Communications Magazine, vol. 56, no. 9, pp. 162-169, Sept. 2018, doi: 10.1109/MCOM.2018.1700659.

[3] S. Gong et al., "Towards smart radio environment for wireless communications via intelligent reflecting surfaces: a comprehensive survey," in IEEE Communications Surveys \& Tutorials, vol. 22, no. 4, pp. 2283-2314, Fourthquarter 2020, doi: 10.1109/COMST.2020.3004197.

[4] Q. Wu and R. Zhang, "Towards smart and reconfigurable environment: intelligent reflecting surface aided wireless network," in IEEE Communications Magazine, vol. 58, no. 1, pp. 106-112, January 2020, doi: 10.1109/MCOM.001.1900107.

[5] W. Yan, X. Yuan, Z.-Q. He, and X. Kuai, "Passive beamforming and information transfer design for reconfigurable intelligent surfaces aided multiuser MIMO systems," in IEEE Journal on Selected Areas in Communications, vol. 38, no. 8, pp. 1793-1808, Aug. 2020, doi: 10.1109/JSAC.2020.3000811.

[6] Q. Cao et al., "Outage Performance Analysis of HARQ-Aided Multi-RIS Systems," 2021 IEEE Wireless Communications and Networking Conference (WCNC), 2021, pp. 1-6, doi: 10.1109/WCNC49053.2021.9417330.

[7] N. K. Kundu and M. R. McKay, "RIS-Assisted MISO Communication: Optimal Beamformers and Performance Analysis," 2020 IEEE Globecom Workshops (GC Wkshps), 2020, pp. 1-6, doi: 10.1109/GCWkshps50303.2020.9367504.

[8] H. U. Rehman, F. Bellili, A. Mezghani, and E. Hossain, "Joint Active and Passive Beamforming Design for IRS-Assisted Multi-User MIMO Systems: A VAMP-Based Approach,” in IEEE Transactions on Communications, vol. 69, no. 10, pp. 6734-6749, Oct. 2021, doi: 10.1109/TCOMM.2021.3094509. 
[9] C. Pan et al., "Multicell MIMO Communications Relying on Intelligent Reflecting Surfaces," in IEEE Transactions on Wireless Communications, vol. 19, no. 8, pp. 5218-5233, Aug. 2020, doi: 10.1109/TWC.2020.2990766.

[10] D.-T. Do, A.-T. Le, T. N. Nguyen, X. Li, and K. M. Rabie, "Joint Impacts of Imperfect CSI and Imperfect SIC in Cognitive RadioAssisted NOMA-V2X Communications," IEEE Access, vol. 8, pp. 128629-128645, 2020, doi: 10.1109/ACCESS.2020.3008788.

[11] A.-T. Le and D.-T. Do, "Implement of multiple access technique by wireless power transfer and relaying network," Bulletin of Electrical Engineering and Informatics (BEEI), vol. 10, no. 2, pp. 793-800, 2021, doi: 10.11591/eei.v10i2.1903.

[12] D.-T. Do, A.-T. Le, and B. M. Lee, "NOMA in Cooperative Underlay Cognitive Radio Networks Under Imperfect SIC," IEEE Access, vol. 8, pp. 86180-86195, 2020, doi: 10.1109/ACCESS.2020.2992660.

[13] C.-B. Le and D.-T. Do, "Employing non-orthogonal multiple access scheme in UAV-based wireless networks," Bulletin of Electrical Engineering and Informatics (BEEI), vol. 10, no. 1, pp. 241-248, 2021, doi: 10.11591/eei.v10i1.2102.

[14] X. Yu, D. Xu, and R. Schober, "Enabling secure wireless communications via intelligent reflecting surfaces," in 2019 IEEE Global Communications Conference (GLOBECOM), 2019, pp. 1-6, doi: 10.1109/GLOBECOM38437.2019.9014322.

[15] C. Huang, A. Zappone, G. C. Alexandropoulos, M. Debbah, and C. Yuen, "Reconfigurable intelligent surfaces for energy efficiency in wireless communication," in IEEE Transactions on Wireless Communications, vol. 18, no. 8, pp. 4157-4170, Aug. 2019, doi: 10.1109/TWC.2019.2922609.

[16] Q. Wu and R. Zhang, "Intelligent reflecting surface enhanced wireless network via joint active and passive beamforming," in IEEE Transactions on Wireless Communications, vol. 18, no. 11, pp. 5394-5409, Nov. 2019, doi: 10.1109/TWC.2019.2936025.

[17] T. Jiang and Y. Shi, "Over-the-Air computation via intelligent reflecting surfaces," 2019 IEEE Global Communications Conference (GLOBECOM), 2019, pp. 1-6, doi: 10.1109/GLOBECOM38437.2019.9013643.

[18] H. Shen, W. Xu, S. Gong, Z. He, and C. Zhao, "Secrecy rate maximization for intelligent reflecting surface assisted multi-antenna communications," in IEEE Communications Letters, vol. 23, no. 9, pp. 1488-1492, Sept. 2019, doi: 10.1109/LCOMM.2019.2924214.

[19] J. Chen, Y.-C. Liang, Y. Pei, and H. Guo, "Intelligent reflecting surface: A programmable wireless environment for physical layer security," in IEEE Access, vol. 7, pp. 82599-82612, 2019, doi: 10.1109/ACCESS.2019.2924034.

[20] M. Cui, G. Zhang, and R. Zhang, "Secure wireless communication via intelligent reflecting surface," in IEEE Wireless Communications Letters, vol. 8, no. 5, pp. 1410-1414, Oct. 2019, doi: 10.1109/LWC.2019.2919685.

[21] W. Yan, X. Yuan, and X. Kuai, "Passive beamforming and information transfer via large intelligent surface," in IEEE Wireless Communications Letters, vol. 9, no. 4, pp. 533-537, April 2020, doi: 10.1109/LWC.2019.2961670.

[22] V. S. Asadchy, M. Albooyeh, S. N. Tcvetkova, A. Díaz-Rubio, Y. Ra'di, and S. A. Tretyakov, "Perfect control of reflection and refraction using spatially dispersive metasurfaces," Physical Review B, vol. 94, no. 7, Aug. 2016, doi: 10.1103/PhysRevB.94.075142.

[23] A. P. Prudnikov, U. A. Bryckov, O. I. Marichev, and G. G. Gould, "More special functions," in Integrals and series, vol. 3, Amsterdam; Paris; New York: Gordon and Breach Science Publishers, 1990.

[24] A. A. Boulogeorgos and A. Alexiou, "Performance analysis of reconfigurable intelligent surface-assisted wireless systems and comparison with relaying," in IEEE Access, vol. 8, pp. 94463-94483, 2020, doi: 10.1109/ACCESS.2020.2995435.

[25] I. S. Gradshteyn and I. M. Ryzhik, Table of Integrals, Series and Products, 6th ed. New York, NY, USA: Academic Press, 2000.

[26] A. M. Mathai and R. K. Saxena, Generalized Hypergeometric Functions with Applications in Statistics and Physical Sciences, in Lecture Notes in Mathematics, vol. 348, 1st ed. Berlin, Germany: Springer-Verlang, 1973, doi: 10.1007/BFb0060468.

[27] A. J. Goldsmith, Wireless Communications. Cambridge, UK: Cambridge University Press, 2005.

\section{BIOGRAPHIES OF AUTHORS}

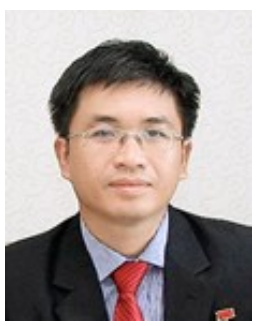

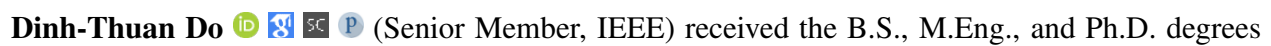
in communications engineering from Vietnam National University (VNU-HCM), in 2003, 2007, and 2013, respectively. His research interests include signal processing in wireless communications networks, cooperative communications, nonorthogonal multiple access, full-duplex transmission, and energy harvesting. He was a recipient of the Golden Globe Award from the Vietnam Ministry of Science and Technology, in 2015 (Top ten excellent young scientists nationwide). He has served as a guest editor for eight prominent SCIE journals. He is currently serving as an associate editor for six journals, including EURASIP Journal on Wireless Communications and Networking, Computer Communications (Elsevier), and KSII Transactions on Internet and Information Systems. He can be contacted at email: dodinhthuan@iuh.edu.vn.

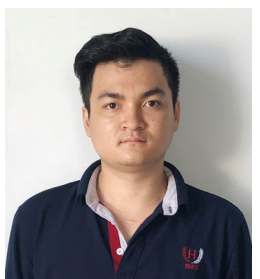

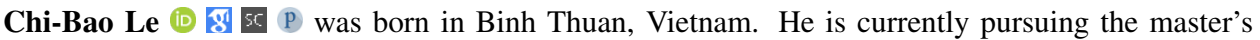
degree in wireless communications. He has worked closely with Dr. Thuan at the Wireless Communications and Signal Processing Research Group, Industrial University of Ho Chi Minh City, Vietnam. His research interests include electronic design, signal processing in wireless communications networks, non-orthogonal multiple access, and physical layer security. He can be contacted at email: lechibao@iuh.edu.vn. 